\title{
Paradiplomacia en tiempos de desastre en el Caribe: un análisis de las islas no soberanas
}

\section{Mayra Vélez Serrano*}

\section{RESUMEN}

En el Caribe coexisten múltiples configuraciones políticas de territorios no-soberanos ligados a las metrópolis de Francia, Estados Unidos, Países Bajos y el Reino Unido. En el 2017, varios de estos territorios fueron devastados por los huracanes María e Irma. Estos eventos pusieron a prueba sus relaciones con las metrópolis y mostraron que la paradiplomacia tiene un rol fundamental en responder a desastres naturales. Mediante el empleo de la técnica del incidente crítico, esta investigación analiza documentos primarios publicados meses posteriores a los huracanes para analizar la variación en el empleo de la paradiplomacia. Muestra que mientras los territorios americanos no utilizaron redes paradiplomáticas, los territorios británicos, como las Islas Vírgenes, agenciaron una mayor participación internacional. Esto les permitió asegurar fondos y ayuda técnica de distintas organizaciones regionales e internacionales. Estas diferencias entre las experiencias paradiplomáticas, requieren una mayor teorización, en la cual se analice el impacto de las

\footnotetext{
* Doctora en ciencia política, con especialidad en relaciones internacionales, política comparada y métodos cuantitativos. Catedrática Asociada en el Departamento de Ciencia Política en la Universidad de Puerto Rico - Recinto de Río Piedras (Puerto Rico). [mayra.velez3@upr.edu]; [https://orcid.org/0000-0003-2339-1182]. En las primeras etapas de investigación, este trabajo fue posible gracias a la ayuda de Nathalie López Del Valle. Gracias a Luis Acevedo Vélez por su ayuda con el manejo de documentos en francés. Previas ediciones de este trabajo fueron presentadas en las conferencias del 2019 del Caribbean Studies Associations en Santa Marta, Colombia, y la Latin American Studies Association en Boston, EE. UU.
}

Recibido: 4 de mayo de 2020 / Modificado: 8 de septiembre de 2020 / Aceptado: 10 de diciembre de 2020

Para citar este artículo:

Vélez Serrano, M. (2021). Paradiplomacia en tiempos de desastre en el Caribe: Un análisis de las islas no soberanas. OASIS, 34, pp. 61-85

Dor: https://doi.org/10.18601/16577558.n34.05 
estructuras versus la agencia de los líderes de cada territorio como posible explicación. Este trabajo descubre entonces la necesidad de un análisis histórico-institucional de la evolución de la paradiplomacia caribeña y la paradiplomacia en general.

Palabras clave: Paradiplomacia, relaciones internacionales del Caribe, desastres naturales, huracán María, huracán Irma.

\section{PARADIPLOMACY IN TIMES OF DISASTER IN THE CARIBBEAN: AN ANALYSIS OF NON-INDEPENDENT ISLANDS}

\section{ABSTRACT}

In the Caribbean region multiple political configurations of non-sovereign territories coexist which link to the metropolises of France, the United States, the Netherlands, and the United Kingdom. In 2017, several of these territories were devastated by the Hurricanes María and Irma. These events put their relations with the metropolises to the test and showed that paradiplomacy has a fundamental role in responding to natural disasters. Using the critical incident method this research analyzes primary documents published months after the hurricanes to analyze the variations in the use of paradiplomacy. It shows that while the American territories did not use paradiplomatic networks, the British territories, such as the Virgin Islands, achieved greater international participation. This allowed them to secure funds and technical assistance from various regional and international organizations. These differences between paradiplomatic experiences require further theorizing, in which the impact of structures versus the agency of the leaders of each territory is analyzed as a possible explanation. This work then discovers the need for a historical-institutional analysis of the evolution of Caribbean paradiplomacy in particular and paradiplomacy in general.

Key words: Paradiplomacy, caribbean international relations, natural disasters, hurricane Maria, hurricane Irma.

\section{INTRODUCCIÓN}

Elaine Stratford describe la ontología de ser isla como

espacios paradójicos: entidades absolutas rodeadas de agua, pero no lo suficientemente grande para ser un continente... (inter)dependientes, identificables; espacios relativos: delimitados pero porosos; aislado, conectado, colonizado y poscolonial... vulnerable a cambios lingüísticos, culturales y ambientales; robustas y capaz de absorber y modificar; paradisiacas, utopías, y distopias... (2003, p. 495).

Estas características hacen el estudio de las islas un área de creatividad, no solo en la conceptualización de las teorías aplicables, usualmente desarrolladas pensando en países de carácter continental, sino en la práctica misma de la creación de políticas públicas. Las islas son, como nos explica Baldacchino, sitios de novedad (2010, p. 14) en los que sus limitaciones geográficas dan paso a una mayor flexibilidad. Además, su aislamiento provee el espacio ideal para la creación de laboratorios (Baldacchino, 
2010, p. 15) políticos, económicos y sociales. ${ }^{1}$ En el mar Caribe, en una pequeña área marítima, coexisten trece islas-territorios que son soberanos y dieciocho territorios dependientes. Cada una refleja grandes diferencias culturales, lingüísticas, étnicas, políticas y económicas.

A pesar de esta diversidad, a las islas caribeñas las unen varios factores. Primero, una historia colonial de explotación, racismo y gran desigualdad económica. Perduran aún los modelos económicos diseñados para la extracción de recursos con poco comercio intrarregional y un capital rentista. No es sorpresivo que las economías caribeñas dependan mayormente del turismo, de los servicios financieros (como el lavado de dinero) y la exportación de productos agrícolas ${ }^{2}$. Además, a las islas caribeñas las une su vulnerabilidad ante desastres naturales tales como terremotos, volcanes y huracanes, sumándose así a sus fragilidades económicas. Según un estudio de Rosajilda Vélez (2019), se estima que los desastres meteorológicos han representado un daño de $1.3 \%$ del producto interno bruto (PIB) de la región ${ }^{3}$.

El Caribe también se caracteriza por la presencia de múltiples territorios subnacionales, o también conocidos como territorios no soberanos, que pertenecen a metrópolis geográficamente distantes. Estas islas existen en un marco político y jurídico impreciso, en el cual no poseen completa soberanía, pero tampoco son una municipalidad definida comúnmente (Baldacchino, 2010, p. 19). Según la sabiduría convencional, tales arreglos permiten que las islas gocen de una relativa autonomía y, a la vez, la ventaja de tener una metrópolis "benevolente" (Baldacchino, 2006, p. 861) y "protectora" (Baldacchino, 2006, p. 856).

Dada esta ambigüedad tanto política como geográfica, las islas-territorios no soberanas del Caribe han aprovechado para agenciar sus propios intereses a través de la paradiplomacia. Esto les ha permitido participar de organizaciones internacionales y regionales, así como extender lazos de cooperación en múltiples áreas funcionales como, por ejemplo, cambio climático, crimen, cultura, turismo, etc. Según Baldacchino, tal autonomía y creatividad diplomática, combinada con la seguridad de la membresía a la metrópolis, provee lo mejor de ambos mundos.

Sin embargo, tales beneficios fueron cuestionados en 2017, luego de la aparición de dos de los huracanes más poderosos que

\footnotetext{
Ejemplo de esta concepción darwiniana de los laboratorios insulares se vio reflejado en la visión de los políticos estadounidenses sobre Puerto Rico. Por ejemplo, el presidente Kennedy se refería a Puerto Rico como "un laboratorio para demostrar a Latinoamérica el desarrollo de la democracia" (89th Congress, 2d Session, 1966, p. 243). Además, el representante Frank T. Bow se refería a Puerto Rico como el único lugar del mundo donde se intentaba "científicamente" permitir la libre empresa, haciendo la isla "nuestro laboratorio líder para la democracia productiva" (Bow, 1951).

$281 \%$ de las exportaciones del Caribe son bienes naturales con bajo valor agregado, mientras que un $4 \%$ consiste en bienes manufacturados de alta tecnología (Vélez, 2019).

3 Según Bertinelli y Strobl (2013, p. 1688), el impacto puede reducir el crecimiento económico alrededor de $1.5 \%$ del рів.
} 
han pasado por la región en décadas, Irma y María. Estos huracanes pasaron con apenas dos semanas de diferencia entre sí, trayendo consigo gran devastación material y humana. Las respuestas de las metrópolis dejaron en entredicho los beneficios económicos y de seguridad a los que alude Baldacchino (2006, 2010). La respuesta del Reino Unido para ayudar a sus territorios fue descrita como "muy lenta", "patética" y que "brillaba por su ausencia” (The Guardian, 2017a; 2017b). Mientras que en Puerto Rico la respuesta fue calificada como "cerca de un genocidio" (The Guardian, 2017c) por las grandes trabas burocráticas requeridas por Washington para aprobar ayuda inmediata.

Esta investigación busca entender cómo las islas-territorios de las metrópolis de Francia, Estados Unidos, Países Bajos y Reino Unido buscaron alternativas paradiplomáticas para responder a la emergencia de los huracanes María e Irma. Se analizan los meses posteriores al paso de estos huracanes por ser momentos críticos, en los que se ponen a prueba las capacidades de los gobiernos locales y la calidad de las relaciones entre las metrópolis y sus territorios. Esta investigación emplea el método del incidente crítico. Este método consiste en describir una situación crítica y las acciones o comportamientos de un actor (en este caso, los gobiernos de estos territorios no soberanos) como repuesta a esta situación.

Para analizar las acciones que los gobiernos locales tomaron luego de estos huracanes, este trabajo utiliza una metodología cualitativa de análisis de documentos oficiales y secundarios. Primero se identificó la fecha en la que los huracanes María e Irma impactaron estos territorios no soberanos. A partir de esta fecha, se buscaron todas las noticias de los periódicos locales relacionadas a dichos huracanes y la respuesta de los gobiernos de estas islas durante un período de 12 meses. En algunas instancias, ciertos periódicos no publicaron nada por un mes o más tiempo, como fue el caso del periódico The Anguillan Newspaper. En estos casos, se obtuvieron noticias en otros periódicos regionales, así como periódicos o noticieros internacionales en español, inglés y francés (ej. The Telegraph y Le Monde). Para analizar la paradiplomacia de estos territorios, esta investigación también recolectó reportes de organizaciones regionales, tales como el Banco de Desarrollo del Caribe, Caricom o la Agencia Caribeña de Seguros de Riesgo de Catástrofe, entre otras.

El estudio de las acciones paradiplomáticas de los territorios no soberanos caribeños no ha contado con el merecido espacio dentro del campo de las relaciones internacionales. Hasta el momento, el estudio de las relaciones internacionales se ha caracterizado por ser Estado-céntrico y eurocéntrico. Incluso la literatura de la paradiplomacia, la cual busca romper con la centralidad del Estado como actor de las relaciones internacionales, tiende también a ser eurocéntrico. El estudio de la paradiplomacia del Caribe no soberano toma importancia no solo porque se concentra en un espacio geográfico muy poco estudiado, sino porque analiza un conjunto de casos que son suigeneris en comparación con los de otros entes políticos practicantes de la paradiplomacia, usualmente estudiados. A diferencia de regiones como Quebec o Cataluña, o de ciudades como Río de Janeiro o Ciudad de México, 
las cuales son partes integrales del Estado, los territorios caribeños no soberanos existen en una ambigüedad legal y política al ser parte de un país, pero sin gozar de una completa incorporación a este.

Aunque este trabajo se limita a describir el contexto y las acciones de estos territorios no soberanos, sirve como base para futuros trabajos que busquen teorizar sobre los factores causales de la paradiplomacia y, sobre todo, descolonizar el estudio de las relaciones internacionales. El descolonizar el estudio de las relaciones internacionales requiere romper con el eurocentrismo que lo domina. Es necesario traer experiencias y conocimiento de otras áreas geográficas (Bilgin, 2016), como en este caso es el Caribe. También requiere romper con las ataduras conceptuales (Wallerstein, 1997) desarrolladas para el estudio del Estado moderno europeo, cuyo afán de universalidad tiende a cegarse ante experiencias y conocimientos externos o de las relaciones coloniales (Maldonado Torres, 2004) que aún prevalecen en el Caribe.

Este escrito está organizado de la siguiente manera: primero, discute las distintas significaciones del término paradiplomacia y precisa cuál se utilizará en esta investigación. Segundo, para entender los marcos legales que regulan la paradiplomacia de estos territorios no-soberanos, se describen los sistemas políticos de estos en relación con sus respectivas metrópolis. Finalmente, se analiza comparativamente la respuesta de la metrópolis ante el desastre natural y cómo los territorios utilizaron sus redes paradiplomáticas para hacer frente inmediato a la emergencia causada por ambos huracanes.

\section{MARCO CONCEPTUAL}

El campo de estudio de la acción internacional de las regiones subnacionales se ha convertido en un fenómeno de gran interés académico en el área de la política comparada y de las relaciones internacionales. Tanto las características, razones y alcances de la acción internacional de los entes subnacionales representan un desafío, no solo al orden mundial westfaliano sino también para los paradigmas dominantes de las relaciones internacionales.

La paradiplomacia describe las acciones de los gobiernos locales o subnacionales, siendo estos últimos definidos como "una expresión genérica utilizada comúnmente para englobar una amplia gama de instituciones, cuya denominación varía de país a país" (Zapata Garesché, 2007, p. 19). Entre los gobiernos locales se incluyen los municipios o ciudades, las áreas metropolitanas, los distritos, departamentos o provincias y las regiones o estados federados. Aparte de la variedad de configuraciones políticas de estos entes subnacionales, las razones para adquirir objetivos y estrategias internacionales son diversas también. Mientras el Estado puede enfocarse en la creación de políticas macro, los gobiernos centrales se han enfocado en la asistencia técnica, transferencia de tecnología, el intercambio de experiencias y la formación de recursos humanos (Zapata Garesché, 2007, p. 24).

Los diferentes actores y objetivos internacionales han hecho que se creen distintos términos para caracterizar la acción internacional de las entidades subestatales. Conceptos como microdiplomacia, protodiplomacia, macrodiplomacia y paradiplomacia surgieron 
para tratar de explicar la acción internacional de los gobiernos locales. Sin embargo, algunos incluyen, dentro del concepto de paradiplomacia, cualquier actividad internacional, incluso de actores no gubernamentales, incluyendo los lobbies internacionales, la industria de los medios de comunicación, movimientos artísticos transculturales, asociaciones científicas e incluso personas destacadas (Aguirre, 2009, p. 196). Esta multiplicidad de actores ha llevado a una plétora de nociones como "diplomacia constituyente" (Kincaid, 1990), "diplomacia multinivel" (Hocking, 1993), "relaciones internacionales subnacionales" (Michelmann, 2009), o "diplomacia subestatal” (Criekemans, 2010).

Este trabajo define paradiplomacia como la acción internacional de los gobiernos subestatales, definidos como "entidad territorial coherente con la capacidad de las tomas de decisiones autoritativas" (Marks, Hooghe y Shaketl, 2008, p. 113), incluyendo regiones, ciudades y territorios no soberanos que buscan adelantar los intereses de sus constituyentes, independientemente de si tal acción "apoya, complementa, corrige, duplica o reta" (Tavares, 2016 , p. 8) las acciones e intereses del gobierno central soberano. Estas acciones incluyen la participación o las relaciones directas con organizaciones internacionales gubernamentales (ej., Caricom, OMc), no gubernamentales (ej., Cruz Roja, Comité Olímpico, etc.) o relaciones con otros gobiernos (ej., regiones, ciudades o gobiernos centrales soberanos).

Esta definición permite incluir la literatura que se ha desarrollado desde la mirada de la acción internacional de ciudades (Tavares, 2013; Vigevani, 2006; Lara Pachecho, 2014;
Zeraoui, 2016; Milani y Ribeiro, 2011; Curtis, 2014), regiones en estados centralizados como España (Villalonga, 1992; Zamorano y Rodríguez, 2014; Criekemans, 2010) o los Estados en federaciones como en Canadá (Balthazar, 1999; Cohn y Smith, 1996; Dyment, 2011; Huijgh, 2010; Lecours, 2010; McHugh, 2015) y Estados Unidos (Cassey, 2014; Kincaid, 1999; Lowenthal, 2009). Igualmente, nos permite analizar la acción internacional de territorios no soberanos y que no son parte integral de un Estado. Es decir, su existencia prevalece entre la ambigüedad de pertenecer a un país, pero sin ser parte integral de este.

Actualmente existen 130 territorios que no tienen soberanía o que tienen un estatus extraterritorial. Muchos de estos territorios son islas que se encuentran en múltiples áreas geográficas, incluyendo el Caribe y el Pacífico. Estas islas no soberanas son conocidas como jurisdicciones insulares subnacionales (Baldacchino, 2006). Aunque carecen de soberanía, tampoco son parte integral del Estado, y poseen diversos grados de autonomía. Sin embargo, tal autonomía coexiste en una relación asimétrica con el Estado central o metrópolis en donde recae el poder político soberano.

El Caribe es una de las regiones en donde más prevalece este tipo de islas o jurisdicciones insulares subnacionales. Por ejemplo, se encuentran los territorios de ultramar de Francia, entre los cuales está Guadalupe, Martinica, St. Bartolomé y St. Martín. También se encuentran los territorios no autónomos del Reino Unido, los cuales son las Islas Caimán, Monserrate, las Islas Vírgenes Británicas (IVB), Anguila, Bermuda, entre otras; mientras que Estados Unidos posee Puerto Rico y las Islas 
Vírgenes de Estados Unidos (Usvi, por sus siglas en inglés).

Estos territorios carecen de soberanía; sin embargo, todos han participado en actividades paradiplomáticas en algún momento dado y con diferentes grados de intensidad. Los motivos, estructura y agencia que explican la paradiplomacia de los territorios no soberanos se diferencian de aquellos que la literatura ha expuesto, analizando los casos de las regiones en Europa o en estados federados. Por ejemplo, las regiones en sistemas federales cuentan con representación entre las estructuras de poder del gobierno central, afectando así los intereses y acciones de los estados. Aun así, muchas regiones adquieren una personalidad internacional para materializar intereses, incluso aquellos contrarios al gobierno central. En el caso de los territorios no soberanos, estos no tienen representación completa (por ejemplo, en las legislaturas o parlamentos) y, por tanto, se puede asumir que la paradiplomacia puede ser más prevalente que en otras entidades subnacionales. A pesar de estas posibilidades, la literatura sobre la paradiplomacia no ha estudiado sistemáticamente la acción internacional de los territorios insulares no soberanos.

\section{LOS SISTEMAS POLIITICOS DEL CARIBE NO SOBERANO}

El Caribe tiene una larga historia de interconexión social y económica entre un mosaico de culturas que se remontan a miles de años antes de la colonización europea (Rodríguez Ramos, 2010; Bright, 2011). Sin embargo, la competencia europea por nuevas tierras coloniales llevó a la ruptura de los lazos que unían a la población indígena. El colonialismo español a finales del siglo xv fue seguido por una ola de otras potencias coloniales como Gran Bretaña, Francia y los Países Bajos a lo largo del siglo XVII. Estas islas se convirtieron en el centro de una explotación económica basada en el monocultivo y la esclavitud.

Aunque podemos encontrar similitudes entre las estructuras políticas y económicas que estos imperios establecieron en el Caribe, también hay diferencias marcadas. Las primeras colonias se basaron en el enfoque tradicional del colonialismo, en el cual los colonos de la metrópoli forjaron una nueva vida en estos territorios, pero su función era proporcionar el control para la extracción de la riqueza. Sin embargo, durante el siglo XIx, el colonialismo se transformó para servir al naciente capital internacional en lugar de a los antiguos Estados monárquicos. El colonialismo pasó del modelo mercantilista extractivo al modelo económico capitalista globalizado. Es en este período que España y Portugal pierden su relevancia en el Caribe, y Estados Unidos se convierte en una potencia imperial en el Caribe cuando toma la posesión de Puerto Rico, las Islas Vírgenes y, por un tiempo, Cuba. Ramos (2016) postula que el colonialismo pasó de significar un modelo en el que los territorios eran colonizados por una casta de residentes de la metrópolis, a la mera administración de territorios y su gente (p. 29). Esta nueva “administración” buscó conciliar la tensión entre la administración de los territorios por parte de la élite de las metrópolis y el interés de mantenerlos dentro de su dominio. El Caribe vio entonces la creación de dependencias, territorios, posesiones, etc., 
todo referido a diferentes arreglos administrativos y sin cambiar su estatus colonial.

En la Tabla 1 se enumeran los nombres de las islas caribeñas no soberanas y sus respectivas metrópolis. A pesar de compartir procesos similares de colonización, existe una gran variación en los sistemas políticos de estas islas. A continuación, se discuten brevemente los sistemas de cada territorio y el grado de autonomía legal que poseen para ejercer política exterior.

Tabla 1

Lista de territorios no soberanos con sus respectivas metrópolis

\begin{tabular}{|l|l|}
\hline \multicolumn{1}{|c|}{ METRÓPOLIS } & \multicolumn{1}{|c|}{ TERRITORIOS } \\
\hline Francia & $\begin{array}{l}\text { Guadalupe } \\
\text { Martinica } \\
\text { San Bartolomé } \\
\text { San Martín }\end{array}$ \\
\hline Países Bajos & $\begin{array}{l}\text { Aruba } \\
\text { Bonaire } \\
\text { Curazao } \\
\text { Saba } \\
\text { San Eustaquio } \\
\text { Sint Maarten }\end{array}$ \\
\hline Reino Unido & $\begin{array}{l}\text { Anguila } \\
\text { Bermuda } \\
\text { Islas Caimán } \\
\text { Turcos \& Caicos } \\
\text { Islas Vírgenes Británicas (IVB) } \\
\text { Montserrat }\end{array}$ \\
\hline Estados Unidos & $\begin{array}{l}\text { Puerto Rico } \\
\text { Islas Vírgenes de los Estados Unidos } \\
\text { (Usvi, por sus siglas en inglés) }\end{array}$ \\
\hline
\end{tabular}

En el caso de las islas francesas existen dos configuraciones políticas en relación con Francia; sin embargo, ambas configuraciones se caracterizan por su integración a la metrópolis. Desde finales del siglo xviıI, Francia adoptó la política de asimilar sus colonias y exten- der los mismos derechos que sus ciudadanos continentales. Este proceso de integración se concretó gracias a la Constitución de la V República, en la cual se estableció la comunidad francesa. Para 1946, Guadalupe, Martinica y Guyana, al igual que las islas de Reunión y Mayotte, se convirtieron en Departamentos de Ultramar (DOM, por sus siglas en francés). Su particularidad es que poseen la misma configuración que los departamentos y regiones que se encuentran en la Francia continental. Por ende, estos reciben los mismos servicios gubernamentales que el resto de Francia. Los habitantes de los DOM están representados por miembros electos al Parlamento, con la diferencia de que se superponen dos estructuras: el departamento y la región. Por lo tanto, solo hay un prefecto, pero dos asambleas: el Consejo General para el departamento y el Consejo Regional. El prefecto es nombrado por Francia y es el representante del Gobierno metropolitano y su rol es encargarse de las relaciones exteriores, defensa y la ley y el orden. Los DOM están sujetos a las leyes francesas, pero con la posibilidad de recurrir a cierta relajación dada su posición geográfica. Como resultado, los DoM gozan de un poco más de autonomía que otros departamentos y regiones de Francia continental.

Por el otro lado, Francia tiene otro nivel de administración conocido como las Colectividades de Ultramar (COM, por sus siglas en francés). En el Caribe, los territorios de San Martín y San Bartolomé tienen la designación de Colectividad de Ultramar desde el 2007. A diferencia de las Dom, cuyo estatus está regido por la Constitución francesa, el estatus político de las colectividades está fi- 
jado por una ley orgánica que especifica sus competencias y las condiciones bajo las cuales las leyes y reglamentos de la metrópolis continental aplicaría. En comparación con las DOM, las сом gozan de mayor autonomía, en especial en asuntos legislativos y fiscales. Sin embargo, incluso dentro de las сом hay diferencias sustanciales. Por ejemplo, las leyes de la Unión Europea y Francia aplican automáticamente a la Colectividades de Ultramar de San Martín, pero no a las Colectividades de San Bartolomé (Sèze, Marchand, \& Bardy, 2012).

En las islas holandesas también hay dos arreglos políticos diferentes: Países Constituyentes y Municipios Especiales. A diferencia de las islas francesas, en las que ambas configuraciones (DOM y COM) se consideran jurisdicciones integradas a la metrópolis, entre las islas holandeses coexisten aquellas que están integradas y las que se definen como países autónomos ${ }^{4}$ (Corbin, 2011). Aruba, Curazao y Sint Maarten son países constituyentes. Según la ley, estas tres islas son países separados dentro del Reino de los Países Bajos. Mientras que Bonaire, Saba y San Eustaquio son municipios especiales, cuyas competencias son similares a las de cualquier municipio de los Países Bajos continentales; es decir, están integrados de forma muy similar a los Departamentos de Ultramar de Francia.

Según la Carta del Reino de los Países Bajos, los asuntos exteriores son responsabilidad del Reino de los Países Bajos. Esto implica que la política exterior del Reino se centra en los intereses de todos los países constituyentes y que las misiones diplomáticas y consulares trabajan para los cuatro países. Aunque una política exterior uniforme es importante para todos los países del Reino, está permitida cierta flexibilidad. Durante la conferencia del Reino de 2011, se acordó que los países constituyentes del Caribe pueden actuar independientemente en sus relaciones con otros países con respecto a sus asuntos autónomos, siempre que se mantenga la unidad de la política exterior del Reino (Departamento de Relaciones Exteriores de Sint Maarten, 2020). Entre los asuntos autónomos en que los países constituyentes pueden tener agencia sobre su política exterior se incluyen las áreas de desarrollo económico, cambio climático, turismo, etc. Según el primer ministro de Curazao, Ivar Asjes, tanto Curazao como los otros países constituyentes del Caribe, han buscado avanzar su desarrollo económico mediante una "mayor integración dentro de la comunidad internacional a través de la participación en organizaciones internacionales y regionales" (Asjes, 2013). En términos generales, los países constituyentes holandeses tienen la potestad de ejercer una paradiplomacia que vele por sus intereses, siempre y cuando no contradiga los intereses de los Países Bajos.

Las islas británicas son los territorios que menos integrados están a su metrópolis en el Caribe. En principio, estos tienen ma-

Según la onu estos se definen como Self-Governing Countries (Corbin, 2011). 
yor autonomía que los territorios franceses y holandeses. Sin embargo, estos tienen una de las deficiencias democráticas más marcadas del Caribe (Corbin, 2011). Ninguno de estos territorios caribeños tiene representación en el Parlamento británico. La mayoría de los territorios británicos se rigen por el Acta de las Indias Occidentales de 1962; con la excepción de Anguila, que se rige por el Acta Anguila de 1980. En estas actas se establece que los territorios no son constitucionalmente parte del Reino Unido. Cada uno tiene una constitución separada, pero todos tienen gobernadores nombrados por la reina Isabel II, que es su jefe de Estado. En general, hay áreas de la política reservadas para el Reino Unido, estas incluyen: defensa, asuntos externos, seguridad interna y servicio público. Mientras tanto, los gobiernos territoriales administran otros aspectos de la política, como la economía, la inmigración y la educación. Hay variación en los poderes y funciones del gobernador nombrado por la corona. Territorios como Bermudas y Gibraltar tienen más autonomía que, por ejemplo, las Islas Turcas y Caicos y Pitcairn (Clegg, 2018). En Bermudas y Gibraltar, el gobernador no preside el gabinete local, pero sí en las Islas Vírgenes. En el caso de Santa Elena, el gobernador también supervisa las finanzas y el transporte marítimo y, en algunos de los territorios del Caribe, el gobernador supervisa aspectos de las finanzas internacionales (Clegg, 2018, p. 150).

Es importante recalcar que este gobernador, nombrado por la corona, no requiere la aprobación de los residentes de los territorios. Aparte de tener poder sobre ciertas áreas reservadas constitucionalmente, también tiene el poder de veto sobre las políticas públicas aprobadas por el parlamento electo por los residentes. Por otro lado, como representante de la corona, al gobernador se le adjudican funciones de política internacional, como es en el caso de la constitución de las Islas Vírgenes Británicas (Corbin, 2011). Según la Constitución de las IVB, se establece explícitamente que el gobernador tiene responsabilidad sobre los asuntos externos en la medida en que se relacionan con cualquier asunto que corresponda a las carteras de los ministros, incluidos la Comunidad del Caribe, la Organización de Estados del Caribe Oriental, la Asociación de Estados del Caribe, la Comisión Económica de las Naciones Unidas para América Latina y el Caribe o cualquier otra organización o institución regional del Caribe; u otros asuntos regionales del Caribe relacionados específicamente con temas de interés o que afecten a las Islas Vírgenes (Artículo 60 de la Constitución Islas Vírgenes Británicas).

Finalmente, los territorios americanos conforman territorios no incorporados $y$, según Corbin (2011), ambos carecen de un gobierno autónomo (self-governing), ya que en ambos se aplican unilateralmente las leyes del Congreso de los Estados Unidos, en el cual ninguno de los territorios tiene representación con voto. Las relaciones entre Puerto Rico y Estados Unidos están reguladas por la Ley 600 de 1950 en la que se crea el Estado Libre Asociado o, en inglés, Commonwealth. Esta establece una autonomía coartada, permitiendo así mantener el control sobre la isla. Por otro lado, las Islas Vírgenes tuvieron un proceso distinto luego de ser compradas a Dinamarca en 1917. Las Islas Vírgenes eran un punto 
estratégico militar (Ramos, 2016), es por esto por lo que Estados Unidos mantuvo control directo hasta 1968 (Virgin Islands Elective Governor Act), cuando los residentes de estas islas pudieron elegir directamente a su gobernador. Sin embargo, las políticas públicas creadas por estos gobiernos locales están sometidas al poder unilateral de las leyes del Congreso Federal y el poder ejecutivo del presidente de los Estados Unidos. De acuerdo con la sección 9 de la Constitución de los Estados Unidos, los estados y, por tanto, los territorios no-incorporados, no pueden ejercer funciones de política exterior. Es decir, los territorios de los Estados Unidos en el Caribe no están incorporados a la metrópolis y tienen menos autonomía, sobre todo en asuntos de política exterior, que sus contrapartes caribeñas.

\section{DESASTRES CLIMÁTICOS DEL CARIBE DE 2017}

En el 2017, la región del Caribe fue abatida por varios huracanes que sentaron récord en términos de intensidad. El huracán Irma fue el noveno en la temporada de huracanes del Atlántico en 2017 y el más poderoso registrado en la historia caribeña, con vientos máximos de aproximadamente 185 millas por hora (mph). Tocó tierra en Barbuda el 6 de septiembre a las 5 de la mañana. Como consecuencia, el 95\% de las casas fueron dañadas o destruidas. La isla terminó inhabitable, y su población (unas 1600 personas) fue evacuada a la isla principal de Antigua. En las Islas Vírgenes Británicas, donde la recuperación de las inundaciones extremas del 7 de agosto de 2017 todavía estaba en marcha, Irma causó daños a casi todas las viviendas, vialidad, puertos, telecomuni- caciones, electricidad, infraestructura e instalaciones críticas, así como la infraestructura del sector turístico se vio significativamente afectada (United Nations Development Program, 2017).

Apenas doce días después del huracán Irma, el huracán María tocó tierra caribeña, sacudiendo a la isla de Dominica el 18 de septiembre del 2017. Toda la población de Dominica (unas 73000 personas) se vio directamente afectada por el huracán María. Más del 90\% de los techos fueron dañados o destruidos. Se interrumpieron los suministros críticos de energía y agua y se destruyeron cultivos enteros. En Sint Maarten, un 91\% de las estructuras y edificios sufrieron daños. En general, entre el 70 y $95 \%$ de las casas sufrieron daños en Anguila, Bahamas, las Islas Turcas y Caicos, las Islas Vírgenes Británicas, Barbuda y Dominica (United Nations Development Program, 2018).

En Puerto Rico, el huracán María se convirtió en uno de los más letales de su historia moderna. Según el Hsiang y Houser (2017) de The Climate Impact Lap, comparado con más de 13000 ciclones en récord desde 1950, María está en la sexta posición en términos del promedio de vientos sentidos por toda un área geográfica. Más de 1.1 millones de casas fueron destruidas, el 95\% de la infraestructura de comunicaciones sufrió daños, el sistema eléctrico colapsó por completo, más de 3 millones de personas vivieron a oscuras por semanas y muchos en áreas rurales y montañosas pasaron casi un año sin electricidad. Se estima que los daños ascendieron a 180 mil millones de dólares en pérdidas (Center for Puerto Rican Studies, 2018). 


\section{RESPUESTA DE LAS METRÓPOLIS AL DESASTRE CAUSADO POR LOS HURACANES IRMA Y MARÍA}

Uno de los problemas más notorios que surgieron por el paso de ambos huracanes fue la pobre y lenta respuesta de las metrópolis. Por ejemplo, el huracán Irma pasó por Anguila el 7 de septiembre del 2019. El barco auxiliar de la Flota Real Británica, Mounts Bay, llegó dos días después, pero solo traía 40 militares y aproximadamente seis toneladas de ayuda. El Reino Unido también despachó la embarcación HMs Ocean ${ }^{5}$, pero este se tardó dos semanas en llegar (House of Commons HC 487). Lamentablemente, mientras el Reino Unido ayudaba a Anguila, las otras islas azotadas por Irma quedaron desprovistas. Estas dos embarcaciones tuvieron que responder a los desastres de dos huracanes consecutivos que golpearon a los territorios británicos. Es decir, tenían que simultáneamente proveer ayuda a islas que estaban aproximadamente a $1600 \mathrm{~km}$ de distancia entre ellas.

El 12 de septiembre de 2017, la primer ministro británica, Theresa May, anunció la ayuda de 25 millones de libras, aparte de los 62 millones que deberían dividirse entre las Islas Vírgenes Británicas, Anguila, Islas Turcas y Caicos y la isla de Dominica (Buchanan 2017). Sin embargo, para abril del 2018, siete meses después del desastre, el Reino Unido no había desembolsado ni un libra del dinero al gobierno de Anguila. El Reino Unido quería nombrar a un director financiero (CFO) como condición para que Anguila recibiera los $£ 60$ millones de ayuda. Por otro lado, había salido a relucir que el secretario de desarrollo, Priti

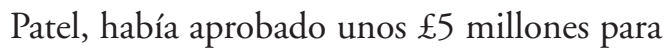
la isla de Dominica (país soberano y excolonia británica), luego de que el Reino Unido había anunciado que no podía dar dinero en efectivo a las Islas Vírgenes Británicas, Anguila o las Islas Turcas y Caicos por considerarlas "países" de alto ingreso (Martin, 2017).

En el caso de las islas holandesas, Sint Maarten fue la más afectada, siendo golpeada tanto por el huracán María como por Irma. A diferencia de las islas británicas, la respuesta del Reino de los Países Bajos fue inmediata. Uno de los problemas apremiantes que sufrió fue la ola de saqueos que se sumó a la inseguridad luego del desastre. Países Bajos inmediatamente desplegó los miembros de las fuerzas armadas y oficiales de la policía para devolver el orden y la seguridad. Durante las primeras semanas luego del desastre, Países Bajos también envió ayuda de emergencia por un valor de $€ 55$ millones a San Martín, Saba y San Eustaquio (Algemene Rekenkamer, 2018). Eventualmente, el gobierno en Holanda aprobó un paquete de ayuda de $€ 550$ millones. Sin embargo, no quiso desembolsar ese dinero directamente a los gobiernos del país de Sint Maarten sin que se cumplieran una serie de condiciones, entre las que se incluía establecer un sistema de

\footnotetext{
5 El hms Ocean era un barco de asalto, antes el portaaviones del Reino Unido y el buque insignia de la flota de la Royal Navy.
} 
monitoreo para supervisar el flujo monetario e implementar medidas anticorrupción. El primer ministro, Williams Marlin, se rehusó a imponer tales medidas, a las que tildó de neocoloniales, pero su Parlamento lo forzó a renunciar luego de emitir un voto de no confianza (DW, 2017).

Este dinero fue depositado en las arcas del Banco Mundial para su administración. Según el estudio de Algemene Rekenkamer (compañía de auditoría independiente), se decidió utilizar los servicios de una organización internacional ya que esto permitiría a los Países Bajos ejercer el control sobre la forma en la que se gastaría el dinero. Es decir, el gobierno holandés podría especificar los términos del mandato, establecer ciertas condiciones y tener un asiento en el Comité Directivo que actuaría como órgano rector del programa. Para julio de 2018, solo se había desembolsado unos $€ 55$ millones de los $€ 550$ millones que los Países Bajos había prometido (The World Bank, 2018).

Puerto Rico, territorio no incorporado de Estados Unidos, tuvo que esperar por más de una semana para que el presidente Donald Trump anunciara el comienzo de las operaciones de ayuda. A pesar de que todos los hospitales de la isla estaban inoperantes por la falta de electricidad, Trump tardó más de una semana en enviar el barco hospital USNS Confort. El $1^{\circ}$ de octubre de 2017, más de diez días después de que el huracán María tocó tierra, Estados Unidos envió un convoy militar para apoyar los esfuerzos de mitigación del desastre. Dado que Estados Unidos controla las fronteras de Puerto Rico, este territorio se rige bajo la ley de la marina mercante conocida como "Ley
Jones de 1917”, en la que se prohíbe la entrada de barcos de bandera extranjera a los puertos de Puerto Rico. Esta prohibición hizo que mucha de la ayuda humanitaria enviada por distintos países tuviera que ir primero a Florida antes de llegar a Puerto Rico. Más tarde, Trump permitió relajar la aplicación de la ley Jones a Puerto Rico por diez días (Paradinas Zorrilla \& Poveda González, 2019), permitiendo así un mayor flujo de carga con ayuda que incluía medicamentos, combustibles, comida y materiales para reparación de la infraestructura de energía, agua y telecomunicaciones. Por otro lado, el Congreso de los Estados Unidos asignó \$ 42 mil millones de ayuda para Puerto Rico, una fracción de los \$92 mil millones de dólares que el Congreso había estimado eran los daños en el territorio. A mediados del 2019 (dos años después del desastre), el gobierno de Puerto Rico solo había podido recibir menos del 33\% de este dinero (Timm, 2019). La falta de ayuda inmediata, así como las limitaciones de obtención de ayuda internacional, provocaron una crisis mayor a la causada como secuela del huracán. Se estima que 2975 personas murieron en los meses posteriores por falta de atención médica y de acceso a electricidad y agua potable (Milken Institute School of Public Health, 2018).

En el caso de los territorios franceses de San Bartolomé y San Martín, estos vieron respuesta inmediata por parte de Francia. A dos días de haber sufrido el embate del huracán Irma, ya Francia había transportado alrededor de 137 toneladas de comida y 500 mil galones de agua para San Martín, en una operación que se describió como "uno de los mayores puentes aéreos desde el comienzo de la Segunda Guerra 
Mundial" (Dalton \& Schechner, 2017). El presidente Macron hizo una visita a las áreas afectadas una semana después del paso del huracán e inmediatamente hizo disponible más de $€ 50$ millones para la respuesta inmediata (Dalton y Schechner, 2017). Estas, al ser parte de la Unión Europea (UE), recibieron ayuda de distintos organismos (Comisión Europea, 2017).

Los problemas de obtención de ayuda por parte de las metrópolis fue un fenómeno generalizado, con la excepción del caso de Francia. Tanto Estados Unidos, Reino Unido y los Países Bajos impusieron fuertes requisitos para el uso de los fondos asignados para responder a la emergencia del desastre. En los casos de Puerto Rico, Sint Maarten y de Anguila se requirió la transferencia de la potestad del manejo y gasto de la ayuda asignada a entes ajenos al gobierno local. Estas condicionalidades demostraron la relación colonial y de subordinación existente a través de todo el Caribe no-soberano.

\section{PARADIPLOMACIA CARIBEÑA ANTE EL CAMBIO CLIMÁTICO Y LOS DESASTRES NATURALES}

Las relaciones internacionales en la región del Caribe coexisten en la presencia de múltiples territorios sin soberanía y dependientes de metrópolis distantes. Dada esta realidad política, la fundación de la Comunidad del Caribe (Caricom) creó mecanismos para que los territorios no soberanos pudieran unirse como miembros asociados. Es decir, la paradiplomacia siempre ha sido parte integral de Caricom (Lacascade \& Laureano-Ortiz, 2019, p. 266). A pesar del fraccionamiento del Caribe, se han alzado regímenes de cooperación, especialmente en el tema de cambio climático y desastres naturales. Laguardia Martínez (2018) explica que en el Caribe existe una densa red de organizaciones formales encargadas de este asunto. Para analizar la amplia red de cooperación paradiplomática, la Tabla 2 enumera aquellas organizaciones caribeñas que tratan el tema de cambio climático y manejos de desastres y los territorios no soberanos que son miembros. Cabe destacar que ninguna de las islas francesas o estadounidenses participan como miembros de estas organizaciones regionales.

Aparte de su participación en organizaciones regionales enfocadas en asuntos de cambio climático y desastres naturales, también vemos que estos territorios participan en múltiples organizaciones regionales. Cabe destacar que existen diferencias en el número de organizaciones a las que estos territorios pertenecen, incluso entre los territorios de una misma metrópolis. De acuerdo con la Tabla 3, las Islas Vírgenes Británicas son las que más actividad paradiplomática tienen, seguida por Anguila. En términos generales, las islas británicas participan en un mayor número de organizaciones regionales e internacionales. Esto se debe, en parte, a que Reino Unido tenía como objetivo la eventual descolonización y la creación de una Federación de las Indias Occidentales en la que pudieran aunar fuerzas para superar sus vulnerabilidades dado su pequeño tamaño. Estos esfuerzos, que comenzaron a mediados del siglo xx, permitieron crear una cultura de cooperación regional. Sin embargo, este proceso de descolonización se detuvo en 1983 y quedaron sin obtener soberanía las islas de Anguila, Islas Caimán, Islas Vírgenes Británi- 
Tabla 2

Participación en organizaciones enfocadas en el cambio climático o mitigación de desastres naturales

\begin{tabular}{|l|l|}
\hline \multicolumn{1}{|c|}{ ORGANIZACIÓN } & $\begin{array}{l}\text { TERRITORIOS MIEMBROS } \\
\text { (COMPLETOS O } \\
\text { ASOCIADOS) }\end{array}$ \\
\hline $\begin{array}{l}\text { Caribbean Meteorological } \\
\text { Organization }\end{array}$ & $\begin{array}{l}\text { Anguila } \\
\text { Bermuda } \\
\text { Islas Caimán } \\
\text { Islas Turcas \& Caicos } \\
\text { Islas Vírgenes Británicas } \\
\text { Montserrat }\end{array}$ \\
\hline $\begin{array}{l}\text { Caribbean Agricultural } \\
\text { Research \& Development } \\
\text { Institute }\end{array}$ & $\begin{array}{l}\text { Islas Caimán } \\
\text { Montserrat }\end{array}$ \\
\hline $\begin{array}{l}\text { Caribbean Disaster } \\
\text { Emergency Management } \\
\text { Agency }\end{array}$ & $\begin{array}{l}\text { Anguila } \\
\text { Monserrat } \\
\text { Islas Turcas \& Caicos } \\
\text { Islas Vírgenes Británicas }\end{array}$ \\
\hline $\begin{array}{l}\text { Caribbean Institute for } \\
\text { Meteorology and Hy- } \\
\text { drology }\end{array}$ & $\begin{array}{l}\text { Anguila } \\
\text { Islas Vírgenes Británicas } \\
\text { Islas Caimán } \\
\text { Monserrat } \\
\text { Islas Turcas \& Caicos } \\
\text { Islas Vírgenes Británicas }\end{array}$ \\
\hline $\begin{array}{l}\text { Caribbean Regional Fishe- } \\
\text { ries Mechanism }\end{array}$ & $\begin{array}{l}\text { Anguila } \\
\text { Islas Turcas \& Caicos } \\
\text { Monserrat }\end{array}$ \\
\hline $\begin{array}{l}\text { Caribbean Public Health } \\
\text { Agency }\end{array}$ & $\begin{array}{l}\text { Anguila } \\
\text { Islas Vírgenes Británicas } \\
\text { Islas Caimanes } \\
\text { Montserrat } \\
\text { Bahamas } \\
\text { Islas Turcas \& Caicos } \\
\text { Aruba } \\
\text { Bermuda } \\
\text { Bonaire } \\
\text { San Eustaquio } \\
\text { Saba } \\
\text { Sint Maarten }\end{array}$ \\
\hline
\end{tabular}

Fuente: Adaptado de Laguardia Martínez (2018, p. 58).

cas y Montserrat (Ramos, 2016, p. 105). Esto puede explicar por qué, en términos generales, las islas británicas tienen mayor actividad paradiplomática.

En el caso de las islas holandesas, parte de su variación en membresía es explicada por la variación en sus sistemas políticos (unas son municipios y los otros países constituyentes del Reino de los Países Bajos). Aruba, Curazao y Sint Maarten son países constituyentes del Reino. A pesar de que, en el sistema político holandés, las relaciones internacionales están controladas por el Reino y no por cada país constituyente, desde la reforma constitucional del 2010 se les permitió agenciar su propia política exterior. Por esta razón, estas islas tienen la mayor cantidad de membresías, tanto como miembros completos o asociados, en organizaciones regionales e internacionales.

Finalmente, cabe destacar los casos de las islas francesas y americanas, los cuales contrastan grandemente en sus relaciones con sus respectivas metrópolis, pero que, al final, tienen muy poca participación en organizaciones intergubernamentales. En el caso de las francesas, como se ha discutido antes, están integradas al Estado francés, mientras que las islas americanas no están integradas, pero su relación con Estados Unidos les prohíbe ejercer relaciones internacionales.

Es evidente que todos los territorios se enfrentaron a severas deficiencias en la repuesta de ayuda de sus respectivas metrópolis. Sin embargo, algunos territorios explotaron sus relaciones paradiplomáticas para obtener ayudas y apoyo de organizaciones internacionales, regionales y organizaciones sin fines de lucro de la sociedad civil, así como de otros países independientes y territorios. La capacidad de utilizar estas líneas paradiplomáticas variaron entre territorios, incluso entre territorios de las mismas metrópolis.

En el caso de Puerto Rico, este estableció relaciones con otros territorios justo 
Tabla 3

Membresía en organizaciones regionales o internacionales

\begin{tabular}{|c|c|c|c|c|c|c|c|c|c|c|}
\hline & омм & OPS & Cepal & AEC & Caricom & Apei & Oeco & BDC & OMT & Total \\
\hline \multicolumn{11}{|l|}{ Británicas } \\
\hline Anguila & $M$ & $M$ & $A$ & & $A$ & & $A$ & $M$ & & 6 \\
\hline Bermuda & $M$ & $M$ & $A$ & & A & & & & & 4 \\
\hline Islas Caimán & $M$ & M & A & & A & & & M & & 5 \\
\hline Islas Turcas y Caicos & $M$ & $M$ & $A$ & & A & & & $M$ & & 5 \\
\hline Islas Vírgenes Británicas & $M$ & $M$ & $\mathrm{~A}$ & & A & A & A & $\mathrm{M}$ & & 7 \\
\hline Montserrat & M & M & $A$ & & M & & & M & & 6 \\
\hline \multicolumn{11}{|l|}{ Holandesas } \\
\hline Aruba & & $M$ & A & $A$ & & $A$ & & & $A$ & 5 \\
\hline Bonaire & & $M$ & & & & A & & & & 2 \\
\hline Curazao & $M$ & $M$ & A & A & & A & & & & 5 \\
\hline Saba & & $M$ & & & & $A$ & & & & 2 \\
\hline Sint Maarten & $M$ & $M$ & A & A & & $A$ & & & & 5 \\
\hline \multicolumn{11}{|l|}{ Francesas } \\
\hline Guadalupe & & $M$ & $A$ & A & & & A & & & 4 \\
\hline Martinica & & $M$ & A & A & & & A & & & 4 \\
\hline San Bartolomé & & & & & & & & & & 0 \\
\hline San Eustaquio & & & & & & A & & & & 1 \\
\hline San Martín & & & & & & & & 0 & & 1 \\
\hline \multicolumn{11}{|l|}{ Americanas } \\
\hline $\begin{array}{l}\text { Islas Vírgenes de } \\
\text { Estados Unidos }\end{array}$ & & & A & & & & & & & 1 \\
\hline Puerto Rico & & $M$ & $A$ & & & $A$ & & & A & 4 \\
\hline
\end{tabular}

Fuente: Adaptado de Laguardia Martínez (2017).

Nota: $\mathrm{M}=$ Miembro, $\mathrm{A}=$ Miembro Asociado, $\mathrm{O}=$ Observador.

Abreviaturas: омм (Organización Meteorológica Mundial); OPS (Organización Panamericana de la Salud); Cepal (Comisión Económica para América Latina); AEC (Asociación de Estados del Caribe); Caricom (Comunidad del Caribe); Apei (Alianza de Pequeños Estados Insulares); Oeco (Organización de Estados Caribeños Orientales); BDC (Banco de Desarrollo del Caribe); OMT (Organización Mundial del Turismo).

luego del impacto del huracán Irma. El gobierno de Puerto Rico estableció centros de ayuda (Noticel, 2017) y recibió refugiados enfermos de las Islas Vírgenes Británicas y San Martín, los cuales se atendieron en los hospitales del país (El Nuevo Día, 2017). Sin embargo, luego del huracán María,
Puerto Rico no estableció contacto con organizaciones regionales o internacionales ni con los gobiernos de otras islas del Caribe. Tanto en los recortes de prensa, informes de organizaciones internacionales y reportes del gobierno se ve una dependencia completa de Estados Unidos. 
Por otro lado, San Bartolomé y San Martín, aparte de algunos grupos civiles como Gustavia Yacht Club (Monacolife, 2018) y organizaciones no gubernamentales como la Cruz Roja (Le Monde, 2017), ninguno de los dos utilizó la paradiplomacia para buscar ayuda inmediata. Por un lado, ambos territorios tienen poca participación en organizaciones regionales e internacionales como se ha mostrado en las Tablas 2 y 3 , mientras que por el otro tampoco se vieron en la necesidad, ya que la respuesta por parte de Francia y la Unión Europea fue inmediata. No obstante, a un año del impacto, San Martín francesa apenas se había reconstruido, dada la burocracia del Estado francés. En una entrevista para el New York Times, Angèle Dormoy, presidenta de la Cámara de Comercio de San Martín decía: "Todo se controla y vuelve a controlar, tres o cuatro veces" (Semple, 2019). Es claro que para estas islas francesas, estar integradas y depender casi enteramente de Francia resultó beneficioso para coordinar la respuesta inmediata al desastre. Sin embargo, la falta de herramientas, flexibilidad y alternativas de financiamiento demostró ser un detrimento a largo plazo.

Por otro lado, Sint Maarten, el territorio holandés, se enfrentó con la desconfianza de Holanda en proveerle los fondos de ayuda directamente a su gobierno, prefiriendo así ser administrado por el Banco Mundial. Esto forzó a Sint Maarten a buscar alternativas para la recuperación y reconstrucción. Por ejemplo, Sint Maarten obtuvo ayuda de la Organización Panamericana de la Salud (Paho, por sus siglas en inglés), de la Comisión Europea y del Departamento para el Desarrollo Internacional del Reino Unido (DFID). Esta obtuvo un paquete de ayuda de US\$148 mil dólares del Fondo Común de Respuesta a Emergencias de la ONU (Cerf, por sus siglas en inglés), sobre US\$300 mil del Programa de las Naciones Unidas para el Desarrollo (UNDP), así como otras donaciones privadas (UNDP Summary Report, 2018). Igualmente, Sint Maarten tiene buenas relaciones con su vecino francés; es decir, los gobiernos de las Sint Maarten y San Martín continuaron sus relaciones diplomáticas. Por ejemplo, en el área de turismo, ambas colaboraron para reconstruir el aeropuerto que está en el lado holandés y así atraer turistas a ambos lados de la isla.

Aunque Sint Maarten pudo aprovechar algunos fondos de organizaciones internacionales, su recuperación fue mayormente propulsada por la inversión del capital privado en el sector del turismo (Semple, 2019). En términos generales, Sint Maarten no pudo aprovechar otras avenidas paradiplomáticas que otros territorios sí obtuvieron, ya que Sint Maarten no es miembro (ver Tabla 3) de la Agencia de Manejo de Emergencias y Desastres en el Caribe (Cdema, por sus siglas en inglés), un organismo de la Caricom, o de la Agencia Caribeña de Seguro de Riesgo de Catástrofe (Ccrif, por sus siglas en inglés).

Las experiencias de estos territorios contrastan, de forma significativa, con los británicos. Luego del impacto de los huracanes, Anguila obtuvo US\$5 millones del Banco de Desarrollo del Caribe (CDB) para la reconstrucción de su infraestructura eléctrica (Caribbean Development Bank, 2017), US\$5.6 millones para asistir en las obligaciones fiscales y US $\$ 9$ millones para implementar un programa de reforma que tuviera como objetivo restaurar 
la sostenibilidad fiscal y mejorar la resiliencia. Igualmente, Anguila recibió las siguientes donaciones: US\$18 millones de la Agencia de Seguro de Riesgo Catastrófico del Caribe (CCRIF), US\$1 millón del Banco Central del Caribe Oriental, US\$200 mil del Banco de Desarrollo del Caribe, US\$50 000 del Gobierno de San Vicente y las Granadinas y US $\$ 400$ mil de la Organización del Caribe Oriental (The Anguillian, 2017).

La paradiplomacia tomó un rol central para las Islas Vírgenes Británicas (IVB) más que para cualquier otro territorio. Estas recibieron en menos de una semana suministros de la $\mathrm{Ca}$ ricom Disaster Relief Unit (CDRU), mientras el equipo de soporte operativo de Caricom (соsт) ayudó con la logística del manejo de la ayuda recibida. Las IVB también recibieron inmediatamente al Equipo de Evaluación Rápida de Necesidades de la Agencia de Manejo de Emergencias y Desastres Naturales (RNAIT-Cdema) para evaluar los daños. La Organización Panamericana de la Salud (ops) y el Programa de las Naciones Unidas para el Desarrollo (Pnud) desplegaron equipos para ayudar en la respuesta al desastre. El organismo ONU Mujeres también llegó a las Islas Vírgenes Británicas para apoyar los programas dirigidos a las mujeres y niños. Finalmente, las Islas Vírgenes Británicas inmediatamente consiguieron el compromiso de ayuda por parte de los Estados participantes de Cdema, y los "socios de desarrollo" también prometieron apoyo, como la Cruz Roja Británica, la Comisión Europea, el gobierno de los Estados Unidos, Asuntos Globales de Canadá, la Federación Internacional de la Cruz Roja, Ocha, la Agencia de Cooperación Internacional de Japón, varias agencias de la ONU, etc. Las Islas Vírgenes Británicas activamente buscaron ayuda de organizaciones regionales, internacionales, de otros países, así como de organizaciones nogubernamentales.

En términos generales, resaltan varios patrones. Primero, los territorios americanos tienen una menor participación en organizaciones regionales $\mathrm{u}$ organizaciones asociadas a asuntos de cambio climático. Esto propició que ambas islas obtuvieran muy poca ayuda por parte de entidades regionales o internacionales. Este fue el caso también para las islas francesas. Sin embargo, las islas francesas obtuvieron una mayor ayuda por parte de Francia y de la Unión Europea, y mucho más rápido que la que obtuvo Puerto Rico desde Estados Unidos. Al otro lado del espectro de la paradiplomacia posdesastre están las islas británicas. Entre Anguila y las Islas Vírgenes Británicas, las últimas tuvieron una paradiplomacia posdesastre más activa. A pesar de que ambas tienen las mismas limitaciones legales en establecer relaciones internacionales, las Islas Vírgenes ha agenciado una mayor participación internacional. Esto le permitió asegurar fondos y ayuda técnica de distintas organizaciones regionales e internacionales. Tanto Anguila como las Islas Vírgenes Británicas tienen una red paradiplomática más densa que las islas francesas o americanas $y$, por tanto, aprovecharon estas relaciones para obtener ayuda cuando el Reino Unido aún brillaba por su ausencia.

El caso de Islas Vírgenes Británicas demostró el beneficio de crear y utilizar ayuda de distintos actores internacionales, incluso en las áreas de preparación y mitigación de desastres naturales. Durante los meses siguientes, luego 
del impacto de ambos huracanes, el gobierno coordinó talleres de capacitación para múltiples sectores de la sociedad civil, incluyendo estudiantes, maestros, líderes comunitarios y religiosos. Estos talleres eran impartidos por expertos provenientes de organizaciones internacionales, no-gubernamentales y de otros países. Por ejemplo, los maestros tomaron talleres con la Agencia Adventista de Desarrollo y Recursos Asistenciales (Adra) y los Fondos Internacionales de Emergencia para la Infancia (Unicef) de las Naciones Unidas. Dueños de empresas tomaron talleres con la Agencia Federal para el Manejo de Emergencias de los Estados Unidos (Fema) y la Cruz Roja (Office of the Deputy Governor, 2018).

El activismo internacional del IVB produjo resultados muy favorables comparado con otros territorios. A nueve meses del paso de estos huracanes, por ejemplo, en el territorio de Puerto Rico aún había un gran segmento de la población sin electricidad y hogares sin techo (El Nuevo Día, 2018). Al mismo tiempo, IVB obtenía, por parte de la ONU, la calificación de excelente en su estado en la recuperación de los desastres. La consultora de Gestión Integral de Desastres (MDL) para las Naciones Unidas (ONU), la señora Rose Ann Smith auditó el Programa de Gestión Integral de Desastres del Territorio para determinar si cumplía con los estándares establecidos por el Marco de Sendai para la Reducción del Riesgo de Desastres y otros marcos regionales y globales (Department of Disaster Management, 2018). Encontró que IVB estaba cumpliendo con todos los estándares, incluso mucho mejor que otras islas del Caribe. Es decir, que no solo logró recuperarse del desastre provocado por ambos huracanes con mucha más rapidez que otros territorios no soberanos del Caribe, sino que también fortaleció su capacidad de prevenir futuros desastres naturales. El caso de IVB apoya el argumento de que la paradiplomacia es una herramienta fundamental de los intereses de los territorios no-soberanos del Caribe.

\section{CONCLUSIÓN}

El Caribe, una región que se destaca por la diversidad de múltiples territorios, los cuales aún existen en un limbo político, que navega en las contradicciones de no ser completamente integrados a sus metrópolis ni completamente soberanos. Su extensión territorial y localización en un área propensa a desastres naturales obliga a repensar las fortalezas de sus estatus políticos.

Del análisis de los sistemas políticos de estos territorios, de la configuración de sus relaciones paradiplomáticas y de las acciones luego del huracán se desprenden varios puntos importantes. Primero, a pesar de que los países constituyentes del Reino de los Países Bajos tiene la potestad de establecer relaciones internacionales, siempre y cuando estas se alineen con la política oficial del Reino, esta potestad no fue aprovechada. Tal aislamiento se vio reflejado en el caso de Sint Maarten el cual dependió mayormente de la ayuda proveniente de Holanda y del sector privado. Al otro lado de la moneda están las islas británicas, las cuales no tienen potestad de hacer política exterior, pero aprovecharon su activismo internacional para obtener ayuda de múltiples organizaciones regionales e internacionales y no depender del Reino Unido. 
Estas diferencias entre las experiencias paradiplomáticas, luego de los huracanes, requieren una mayor teorización, en la cual se analice el impacto de las estructuras versus la agencia de los líderes de cada territorio como posible explicación. Como hipótesis tentativa, se puede deducir que los marcos legales no son condición suficiente, aunque sí necesaria, para explicar la variación en la actividad paradiplomática en el Caribe. Este trabajo descubre entonces la necesidad de un análisis históricoinstitucional de la evolución de la paradiplomacia caribeña y la paradiplomacia en general.

\section{REFERENCIAS}

Aguirre, I. (2009). Making sense of paradiplomacy? An intertextual enquiry about a concept in search. Paradiplomacy in action: The foreign relations of subnational governments, 9(1), 185-209.

Alders, A. A. (2015). Obstacles to 'good governance' in the Dutch Caribbean: Colonial-and postcolonial development in Aruba and Sint Maarten (Tesina sin publicar) Utrecht University.

Algemene Rekenkamer. (2018). Focus op de Nederlandse bijdrage aan de wederopbouw van SintMaarten (Focus on the Dutch contribution to the reconstruction of Sint Maarten). Disponible en https://english.rekenkamer.nl/publications/reports/2018/12/13/focus-on-thenetherlands\%E2\%80\%99-contribution-to-thereconstruction-of-sint-maarten

Baldacchino, G. (2006). Innovative development strategies from non-sovereign island jurisdictions? A global review of economic policy and governance practices. World Development, 34(5), 852-867.
Baldacchino, G. (2010). Island enclaves: Offshoring strategies, creative governance, and subnational island jurisdictions. Montreal: McGill-Queen's Press.

Balthazar, L. (1999). The Quebec experience: Success or failure? Regional \& Federal Studies, 9(1), 153-169.

Bertinelli, L. \& Strobl, E. (2013). Quantifying the local economic growth impact of hurricane strikes: An analysis from outer space for the Caribbean. Journal of Applied Meteorology and Climatology, 52(8), 1688-1697.

Bilgin, P. (2016). How to remedy Eurocentrism in IR? A complement and a challenge for the global transformation. International Theory, 8(3), 492-501.

Bow, F. T. (1951). The Puerto Rican manifesto - The answer to Eastern and Western Collectivism. Remarks. Congressional Record. Proceeding and Debates of the 82nd Congress. First Session, vol. 97, Part 13.

Bright, A. J. (2011). Blood is thicker than water: Amerindian intra-and inter-insular relationships and social organization in the pre-Colonial Windward Islands. Leiden: Sidestone Press.

Buchanan, R. T. (13/09/2017). Theresa May just announced an extra $£ 25$ million for hurricane Irma victims. BuzzFeed News. Disponible en https:// www.buzzfeed.com/rosebuchanan/theresa-mayjust-announced-an-extra-ps25-million-for

Caribbean Development Bank. (2017). CDB approves funding to restore electricity and build climate resilience in Anguilla. Disponible en https://www. caribank.org/newsroom/news-and-events/cdbapproves-funding-restore-electricity-and-buildclimate-resilience-anguilla

Cassey, A. J. (2014). The location of US States' Overseas Offices. Review of International Economics, 22(2), 310-325. Disponible en https://doi.org/10.1111/ roie. 12109

Center for Puerto Rican Studies. (03/2018). Puerto Rico post-Maria report $[\mathrm{PDF}]$. Disponible en https:// 
centropr.hunter.cuny.edu/sites/default/files/PDF/ puerto_rico_post_maria-2018-final.pdf

Clegg, P. (2018). The United Kingdom and its Overseas Territories: No longer a benevolent patron? Small States \& Territories, 1(2), 149-168.

Cohn, T. H. \& Smith, P. J. (1996). Subnational governments as international actors: Constituent diplomacy in British Columbia and the Pacific Northwest. BC Studies: The British Columbian Quarterly, (110), 25-59. https://doi.org/10.14288/ bcs.v0i110.1339.

Comisión Europea. (13/12/2017). Solidarity Fund provides initial financial aid for Saint-Martin and Guadeloupe. Disponible en https:// ec.europa.eu/regional_policy/en/newsroom/ news/2017/12/13-12-2017-solidarity-fundprovides-initial-financial-aid-for-saint-martinand-guadeloupe

Conlan, T. J. (2010). From new federalism to devolution: Twenty-five years of intergovernmental reform [eBook edition]. Brookings Institution Press.

Constitution of the British Virgin Islands. (2007). [PDF]. Disponible en https://bvi.gov.vg/sites/default/ files/constitution.pdf

Constitution of the United States. (s.f.). Disponible en https://www.usconstitution.net/const.txt

Corbin, C. G. (2011). Self-Governance deficits in Caribbean dependency and autonomous models, Overseas Territories Report, 10(2). Recuperado 20/02/2018 de https://www.alainet.org/en/active/45629

Cornago, N. (2018). Paradiplomacy and protodiplomacy. En G. Martel (Ed.), The Encyclopedia of diplomacy (pp. 1-8). Hoboken, NJ: Wiley.

Criekemans, D. (2010). Regional sub-state diplomacy from a comparative perspective: Quebec, Scotland, Bavaria, Catalonia, Wallonia and Flanders. The Hague Journal of Diplomacy, 5(1-
2), 37-64. https://doi.org/10.1163/18711911 0X12574289877489

Curtis, S. (Ed.). (2014). The power of cities in international relations. Routledge.

Dalton, M. \& Schechner, S. (12/09/2017). France carries out massive aid mission in Caribbean. The Wall Street Journal. Disponible en https://www.wsj. com/articles/france-plans-massive-aid-missionfor-st-martin- 1505243860

Departamento de Relaciones Extranjeras de Sint Maarten. (2020). Disponible en http://www.sintmaartengov.org/government/AZ/Department-ofForeign-Relations/Pages/default.aspx

Department of Disaster Management. (25/07/2018). $B V I$ rated in excellent standing on disaster recovery. [Comunicado de Prensa]. Disponible en http:// www.bvi.gov.vg/media-centre/bvi-rated-excellent-standing-disaster-recovery

Duchacek, I. D. (1986). International competence of subnational governments: Borderlines and beyond. En O. J. Martinez (Ed.), Across boundaries: Transborder interaction in comparative perspective (pp. 11-28). El Paso, TX: Texas Western Press.

DW News. (24/11/2017). Dutch Saint Martin's leader quits over Hurricane Irma aid controversy. Disponible en https://p.dw.com/p/2oEYP.

El Nuevo Día. (11/09/2017). Puerto Rico es centro de ayuda para islas vecinas arrasadas por Irma. Disponible en https:/www.elnuevodia.com/noticias/ locales/nota/puertoricoescentrodeayudaparaislasvecinasarrasadasporirma-2356832/

El Nuevo Día. (20/06/2018). Muchas casas lucen sin techo al inicio de la temporada de huracanes. Disponible en https://www.elnuevodia.com/ noticias/locales/notas/muchas-casas-lucen-sintecho-al-inicio-de-la-temporada-de-huracanes/ Garesché, E. D. Z. (2007). Manual práctico para internacionalizar la ciudad: Guía para la acción 
exterior de los gobiernos locales y la cooperación descentralizada Unión Europea-América Latina [pDF file]. Disponible en https://www.ritimo. org/Manual-practico-para-internacionalizar-ala-ciudad-Guia-para-la-accion

Hintjens, H. \& Hodge, D. (2012). The UK Caribbean Overseas Territories: Governing unruliness amidst the extra-territorial EU. Commonwealth \& Comparative Politics, 50(2), 190-225.

Hocking, B. (1993) Localizing foreign policy: Non-central governments and mutilayered Diplomacy. New York: Mcmillan Press.

House of Commons. (31/10/2017). Oral evidence: The UK's response to Hurricane Irma (International Development Committee HC 487). Disponible en http://data.parliament.uk/writtenevidence/committeeevidence.svc/evidencedocument/ international-development-committee/the-uksresponse-to-hurricane-irma/oral/72706.html

Hsiang, S. \& Houser, T. (29/09/2017). Don't let Puerto Rico fall into an economic abyss. The New York Times. Disponible en https://www.nytimes. com/2017/09/29/opinion/puerto-rico-hurricane-maria.html?_r=0

Huijgh, E. (2010). The public diplomacy of federated entities: Examining the Quebec model. En D. Criekemans (Ed.), Regional sub-state diplomacy today (pp. 125-150). Leiden: Martinus Nijhoff Publishers.

Keohane, R.O. \& Nye, J. (1989). Power and interdependence ( $2^{\text {nd }}$ ed.). Glenview, IL: Scott, Foresman and Company.

Kissinger, H. (1975). A new national partnership. (Discurso del Secretario de Estado Henry A. Kissinger en Los Ángeles, CA. el 24/01/1975.) Bureau of Public Affairs, Office of Media Services.

Kincaid, J. (1990). Constituent diplomacy in federal polities and the nation state: Conflict and coo- peration. En H. J. Michelmann and P. Soldatos (Eds.), Federalism and international relations: The role of subnational unit. Oxford: Clarendon Press Publication.

Lacascade, J.Y. \& Laureano Ortiz, R. (2019). Caribbean climate paradiplomacy of the European Union's Overseas Regions and Territories: Towards an insertion in region wide cooperation within the context of the EU-Celac partnership. En E. Dubesset \& C. Quenan (Eds.), La Caraïbe dans le partenariat stratégique euro-latino-américain UE-Celac. EU-LAC Foundation.

Laguardia Martínez, J. (11-12/2017). Los territorios no independientes del Caribe: Notas sobre su actualidad y circunstancia. Revista CariCen 5., 46-62. Laguardia Martínez, J. (2018). La gobernanza ambiental en el Caribe de la Caricom para la gestión del cambio climático. Entretextos 9 (27), 51-71. Disponible en http://investigacion.politicas. unam.mx/caricen/wp-content/uploads/caricen5/ caricen5_2_1.pdf

Lara Pacheco, R. F. (2014). Una tipología para el estudio de la proyección de las ciudades en el medio internacional. En A. B. Rodríguez \& Ó. C. Montellano (Eds.), La construcción del futuro: los retos de las ciencias sociales en México. Memorias del 4 Congreso Nacional de Ciencias Sociales (pp. 293-310). Cesmeca.

Lecours, A. (2010). Canadian federalism and foreign relations: Quebec and Alberta. En F. Requejo (Ed.), Foreign policy of constituent units at the beginning of 21st century (pp. 29-41). Institut d'Estudis Autonòmics.

Lecours, A. \& Moreno, L. (2003). Paradiplomacy: A nation-building strategy? A reference to the Basque Country. En A. Gagnon, M. Guibernau, \& F. Rocher (Eds.), The conditions of diversity in 
multinational democracies (pp. 267-292). Institute for Research on Public Policy.

Le Monde. (13/09/2017). Irma: Emmanuel Macron promet un mécanisme d'aide financière "d'urgence» pour les sinistrés. Disponible en https://www. lemonde.fr/climat/article/2017/09/13/apressaint-martin-emmanuel-macron-attendu-a-saintbarthelemy_5184989_1652612.html

Lowenthal, A. F. (2009). Global California: rising to the cosmopolitan challenge. Stanford University Press.

Maldonado-Torres, N. (2004). The topology of being and the geopolitics of knowledge: Modernity, empire, coloniality. City, 8(1), 29-56.

Marks, G., Hooghe, L. \& Schakel, A. H. (2008). Measuring regional authority. Regional \& $\mathrm{Fe}$ deral Studies, 18(2-3), 111-121. https://doi. org/10.1080/13597560801979464.

Martin, D. (25/09/2017). The hurricane force farce continues: British island received nothing from the foreign aid budget while non-British island received $£ 5$ million. Daily Mail. Disponible en https:// www.dailymail.co.uk/news/article-4915956/ British-island-received-foreign-aid-budget.html

McHugh, J. T. (2015). Paradiplomacy, protodiplomacy and the foreign policy aspirations of Quebec and other Canadian provinces. Canadian Foreign Policy Journal, 21(3), 238-256. https://doi.org/10. 1080/11926422.2015.1031261.

Milani, C. R. S. \& Ribeiro, M. C. M. (2011). International relations and the paradiplomacy of Brazilian cities: crafting the concept of local international management. BAR - Brazilian Administration Review, 8(1), 21-36. https://doi.org/10.1590/ S1807-76922011000100003.

Milani, Z. \& Villar, F. R. C. (2016). La paradiplomacia de la ciudad. Una estrategia de desarrollo urbano. Revista del CLAD Reforma y Democracia, (65), 225-242.
Michelmann, H. (2009). Foreign relations in federal countries. McGill-Queen's Press.

Milken Institute School of Public Health. (2018). Ascertainment of the estimated excess mortality from hurricane Maria in Puerto Rico. The George Washington University. https://bit.ly/3lmHjW7 Monacolife. (01/01/2018). Gustavia Yacht Club distributes $€ 190,000$ of Irma relief fund. Disponible en https://monacolife.net/gustavia-yacht-clubdistributes-e190000-of-irma-relief-fund/

Noticel (11/09/2017). HIMA and Verdanza in the 'Eye' of Puerto Rican kindness and solidarity (video) Disponible en https://www.noticel.com/huracanes/ el-tiempo/english/la-calle/weather/20171017/ hima-and-verdanza-in-the-eye-of-puerto-ricankindness-and-solidarity-vid/

Office of the Deputy Governor. (12/07/2018). Residents receive training to enhance their state of readiness [Comunicado de Prensa]. Disponible en http:// www.bvi.gov.vg/media-centre/residents-receivetraining-enhance-their-state-readiness

Paradinas Zorrilla, R., \& Poveda González, M.E. (2019). Momento crucial para Puerto Rico: Una economía desconocida integrada en el mercado estadounidense. Boletín Económico de Ice (3108), 3-18.

Rekenkamer, A. (2018). Focus on the Dutch Contribution to the reconstruction of Sint Maarten [PDF]. Disponible en https://english.rekenkamer.nl/ publications/reports/2018/12/13/focus-on-thenetherlands\%E2\%80\%99-contribution-to-thereconstruction-of-sint-maarten

Ramos, A.G. (2016). Islas Migajas: Los paises no independientes del Caribe contemporáneo. San Juan, PR: Travesier \& Leduc Editores.

Rodríguez Ramos, R. (2010). What is the Caribbean? An archaeological perspective. Journal of Caribbean Archaeology, 3, 19-51. 
Sèze, N.; Marchand, A. \& Bardy, R. (2012). French overseas territories and the euro. Banque de France: Quarterly Selection of Articles (24), 101-124.

Setzer, J. \& Anderton, K. (2019). Subnational leaders and diplomacy. Oxford research encyclopedia: Diplomacy, environment, foreign policy. Disponible en https://doi.org/10.1093/acrefore/9780190846626.013.504

Stratford, E. (2003). Flows and boundaries: small island discourses and the challenge of sustainability, community and local environments. Local Environment, 8(5), 495-499. https://doi.org/10.108 0/1354983032000143653.

Tavares, R. (09/10/2013). Foreign Policy Goes Local: How Globalization Made Sao Paulo into a Diplomatic Power. Foreign Affairs. Disponible en https://www.foreignaffairs.com/articles/southamerica/2013-10-09/foreign-policy-goes-local.

Tavares, R. (2016). Paradiplomacy: Cities and states as global players. New York: Oxford University Press.

The Anguillian. (23/10/2017). Anguilla to lose millions from closure of Hotels Wants UK aid up to $\$ 16.5$ million or more. Disponible en http:// theanguillian.com/2017/10/anguilla-to-losemillions-from-closure-of-hotels-wants-uk-aidup-to-16-5-million-or-more/

The Bureau for Latin America and the Caribbean. (07/01/2019). Hurricanes Irma and Maria: One year on. Disponible en https://www.latinamerica.undp.org/content/rblac/en/home/library/ crisis_prevention_and_recovery/hurricanes-irmaand-maria--one-year-on.html

The Guardian. (06/09/2017a). UK government accused of pathetic lack of help for Anguillians hit by Irma. Disponible en https://www.theguardian. com/world/2017/sep/06/government-accusedof-disgraceful-lack-of-aid-for-anguillans-hitby-irma
The Guardian. (01/05/2017b). UK releases $£ 32 \mathrm{~m}$ in Hurricane Irma aid after complaints over initial response. Disponible en https://www.theguardian. com/world/2017/sep/07/ukcould-send-furthership-to-overseas-territories-hit-by-hurricane

The Guardian. (22/09/2017c). 'Close to genocide': San Juan mayor's dire appeal to US for Puerto Rico relief. Disponible en https://www.theguardian. com/world/2017/sep/29/san-juan-mayor-criticizes-us-officials-for-calling-puerto-rico-reliefeffort-good-news

Semple, K. (07/10/2019). After a Caribbean hurricane, the battle is where, or even whether, to rebuild. The New York Times. Disponible en https:// www.nytimes.com/2019/10/07/world/americas/ hurricane-irma-saint-martin.html

The World Bank. (12/07/2018). First US\$55M grant from the Sint Maarten trust fund for recovery \& resilience [Comunicado de Prensa]. Disponible en https://www.worldbank.org/en/news/pressrelease/2018/07/12/first-us55m-grant-from-thesint-maarten-trust-fund-for-recovery-resilience

Timm, J. C. (18/07/2019). Fact check: Trump says Puerto Rico got $\$ 92$ billion. They've seen only a fraction. NBC News. Disponible en https:// www.nbcnews.com/politics/donald-trump/factcheck-trump-says-puerto-rico-got-92-billionthey-n1031276

United Nations Development Program. (2018). From early recovery to long-term resilience in the Caribbean. Disponible en https://www.bb.undp. org/content/dam/barbados/docs/Publications/ undp_bb_Undp-Recovery-Programme $\% 20$ 2\%20oct\%20WEB.pdf

United Nations Development Program. (2017). Regional overview: impact of hurricanes Irma and Maria. Disponible en https://www.latinamerica.undp. $\mathrm{org} /$ content/rblac/en/home/library/environ- 
ment_energy/regional-overview--impact-ofhurricanes-irma-and-maria.html

Vadell, A. V. (1992). Las relaciones institucionales entre la Comunidad Autónoma de Cataluña y América Latina. Barcelona Centre for International Affairs Magazine, (23-24), 291-322. https://doi. org/27918

Vélez, R. (04/2019). Las economías de la zona del Caribe en el contexto de la revolución tecnológica. Études Caribéennes 42, 1-16. https://doi.org/10.4000/ etudescaribeennes. 15177.

Vigevani, T. (2006). Problemas para a atividade internacional das unidades subnacionais: estados e municípios brasileiros. Revista Brasileira de Ciências Sociais, 21(62), 127-139. https://doi.org/10.1590/ S0102-69092006000300010

Wall Street Journal. (12/09/2017). France Carries Out Massive Aid Mission in Caribbean. Disponible en https://www.wsj.com/articles/france-plansmassive-aid-mission-for-st-martin-1505243860 Wallerstein I. (1997). Eurocentrism and its avatars: The dilemmas of social science. Sociological Bulletin, 46(1), 21-39.

Zamorano, M. M. \& Rodríguez A. M. (2014). The cultural paradiplomacy of Barcelona since the 1980s: Understanding transformations in local cultural paradiplomacy. International Journal of Cultural Policy 21(5), 554-576. https://doi.org/10.1080/ 10286632.2014.943752.

Zapata Garesché, E. D. (2007). Manual práctico para internacionalizar la ciudad: Guía para la acción exterior de los gobiernos locales y la cooperación descentralizada Unión Europea-América Latina, vol. 1. Diputación de Barcelona. 